\title{
Different Times of Origin of Choline Acetyltransferase- and Somatostatin-Immunoreactive Neurons in the Rat Striatum
}

\author{
Kazue Semba, Steven R. Vincent, and Hans C. Fibiger \\ Division of Neurological Sciences, Department of Psychiatry, University of British Columbia, Vancouver, B.C. V6T 2A1, \\ Canada
}

\begin{abstract}
Two populations of aspiny interneurons have been identified in the mammalian striatum, one cholinergic and the other using the neuropeptide somatostatin as a neurotransmitter. The times at which these 2 cell populations undergo their final mitosis were studied by injecting tritiated thymidine into timed pregnant rats and then processing the brains of the progeny as young adults for immunohistochemistry with monoclonal antibodies to choline acetyltransferase and somatostatin followed by autoradiography. Choline acetyltransferase-immunoreactive neurons became postmitotic in a caudal-to-rostral gradient; the occurrence of final mitosis was maximal on embryonic day (E) 12 at the most caudal level and on E15 at the most rostral. A more subtle lateralto-medial gradient was also observed in the precommissural striatum. In contrast, no obvious gradients were seen with somatostatin-immunoreactive neurons; regardless of their location within the striatum, these neurons underwent their final mitosis on days E15-16, towards the end of cholinergic neurogenesis. These results indicate that although both cholinergic and somatostatin-containing cells represent interneuronal populations in the striatum, they display distinctly different spatiotemporal patterns of neurogenesis.
\end{abstract}

In the mammalian striatum, the vast majority of neurons are medium-sized spiny cells, which are projection neurons containing GABA, substance $P$, and endogeneous opioids (Graybiel and Ragsdale, 1983; Parent, 1986; Semba et al., 1987). The remaining ncurons arc mostly interneurons, and of these the large aspiny neurons are believed to be cholinergic (Fibiger, 1982). Somatostatin (SOM) is present in a subpopulation of medium-sized aspiny interneurons, and these neurons also contain neuropeptide Y (Vincent et al., 1982; Smith and Parent, 1986), as well as NADPH-diaphorase enzyme activity (Vincent et al., 1983a, b). Both cholinergic (Satoh et al., 1983; Bolam et al., 1984; Phelps et al., 1985) and SOM-containing interneurons (DiFiglia and Aronin, 1982, 1984; Takagi et al., 1983) receive occasional synaptic contacts on the soma and dendrites; some of the axonal terminals presynaptic to cholinergic neurons contain substance P (Bolam et al., 1986) or tyrosine hydroxylase (Kubota et al., 1987). The axons of the cholinergic (Bolam et

\footnotetext{
Received Jan. 21, 1988; revised Mar. 7, 1988; accepted Mar. 8, 1988

We thank F. Eckenstein and J. C. Brown for generous gifts of monoclonal antibodies to choline acetyltransferase and somatostatin, respectively, and S. At madja and N. Lee for excellent technical assistance. Supported by the Medical Research Council of Canada. S.R.V. is an MRC Scholar.

Correspondence should be addressed to Kazue Semba, Ph.D., at the ahove address.

Copyright (c) 1988 Society for Neuroscience $0270-6474 / 88 / 103937-08 \$ 02.00 / 0$
}

al., 1984; Phelps et al., 1985) and SOM-containing interneurons (DiFiglia and Aronin, 1982, 1984; Takagi et al., 1983) appear to make synapses with the somata and dendrites of striatal neurons, including medium spiny neurons. Thus, these different types of neuron form a complex neuronal circuitry to process afferent information from the cortex, thalamus, and substantia nigra for output directed to the pallidum and substantia nigra (Semba et al., 1987).

Little is known about the manner in which the neuronal circuitry in the striatum develops during ontogeny. The time of final mitosis of developing striatal neurons has been studied by using ${ }^{3} \mathrm{H}$-thymidine autoradiography in Nissl-stained material in the rat (Fentress et al., 1981; Bayer, 1984; Marchand and Lajoie, 1986; van der Kooy and Fishell, 1987), mouse (Angevine and McConnell, 1974; Fentress et al., 1981), Chinese hamster (ten Donkelaar and Dederen, 1979), rabbit (Fernández et al., 1979), cat (Graybiel and Hickey, 1982), and primate (Brand and Rakic, 1979). However, none of these studies took into account the neurochemical heterogeneity of striatal neurons. In the present study, ${ }^{3} \mathrm{H}$-thymidine autoradiography was combined with immunohistochemistry using monoclonal antibodies to choline acetyltransferase (ChAT) and SOM to study the time of final mitosis of 2 major interneuronal populations in the striatum. Preliminary results have been reported (Semba et al., 1986; Fibiger et al., 1987).

\section{Materials and Methods}

The experimental procedures regarding animal breeding, immunohistochemistry, and autoradiography have been described previously in detail (Semba and Fibiger, 1988), and only a brief description of these techniques is provided here. Procedural details concerning the data analyses specific to striatal neurons are fully described.

Each of 9 precisely timed $( \pm 3 \mathrm{hr})$ pregnant Sprague-Dawley rats received a single pulse injection of ${ }^{3} \mathrm{H}$-thymidine $(7 \mu \mathrm{Ci} / \mathrm{gm}$, i.p., $6.7 \mathrm{Ci} /$ mmol, NET-027, New England Nuclear) on one day from embryonic day (E) 10 to 18 . The day of conception was designated as $\mathrm{E} 0$. The pregnant rats were allowed to deliver, and when their progeny were about 6 weeks old, $2-4$ males for each day of ${ }^{3} \mathrm{H}$-thymidine injection were randomly selected. Under deep anesthesia, these rats were perfused with a buffered paraformaldehyde fixative. Following postfixation and cryoprotection, the brains were cut into $30 \mu \mathrm{m}$ coronal sections, which were then processed for immunohistochemistry followed by autoradiography using Kodak NTB-2 with 3 weeks exposure at $4^{\circ} \mathrm{C}$. Immunohistochemistry was conducted using a rat monoclonal antibody to ChAT (diluted 1:100 or 1:200) or a mouse monoclonal antibody to SOM (Ab8 diluted to $5 \mu \mathrm{g} / \mathrm{ml}$ ). These antibodies were visualized using the avidin-biotin-peroxidase complex method (Hsu et al., 1981) and a standard diaminobenzidine method. The specificity of these antibodies, as well as their use in immunohistochemical studies of the rat brain, has been documented (ChAT: Eckenstein and Thoenen, 1982; SOM: Vincent et al., 1985).

For data analysis, a set of 6 coronal sections encompassing the entire 

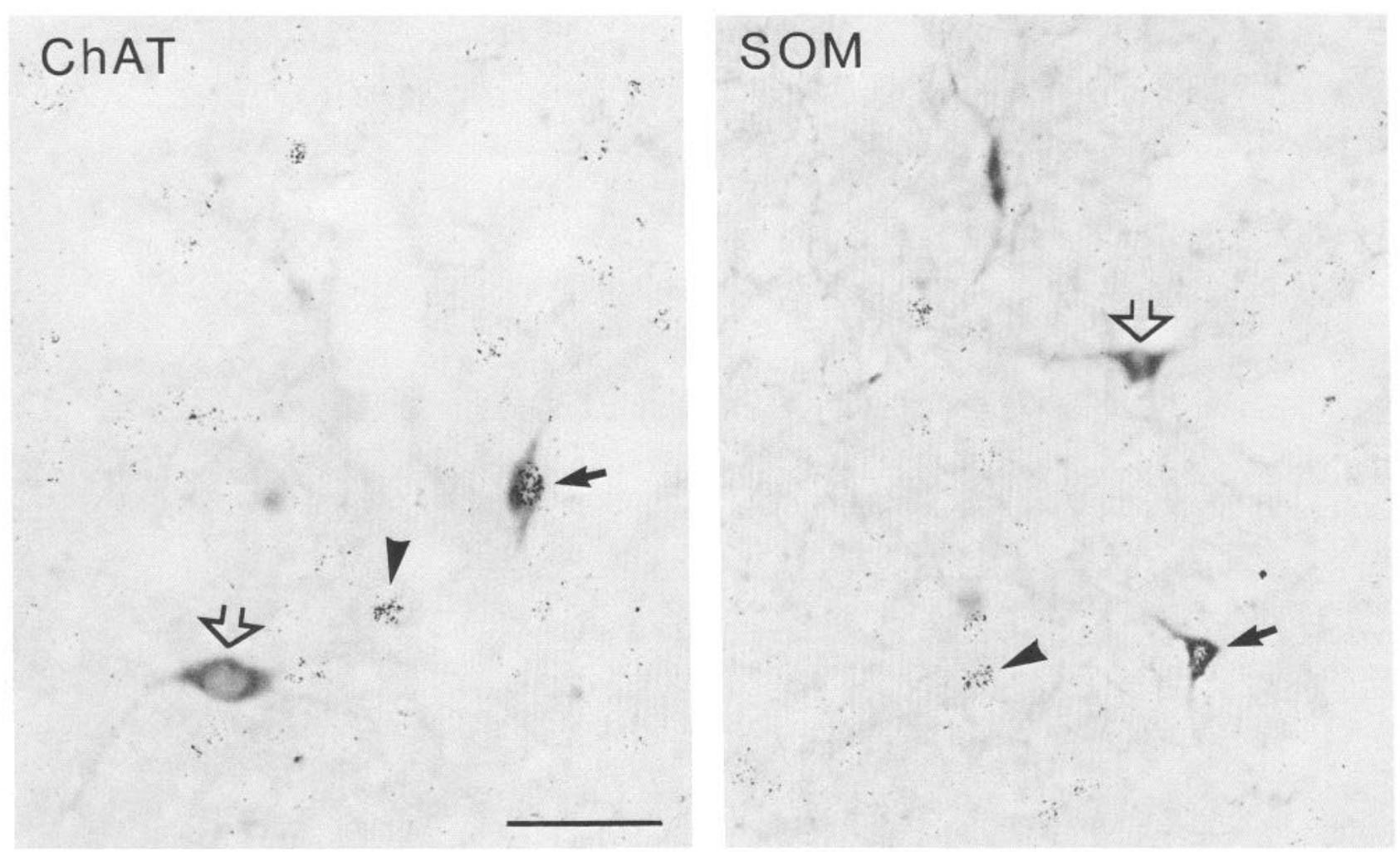

Figure 1. Examples of striatal neurons double-labeled for ChAT or SOM and ${ }^{3} \mathrm{H}$-thymidine (arrows), neurons labeled with immunoreactivity alone (open arrows), and neurons labeled with ${ }^{3} \mathrm{H}$-thymidine alone (arrowheads). Micrographs are from rats exposed to ${ }^{3} \mathrm{H}$-thymidine in utero on E14 (ChAT) and E15 (SOM). Scale bar, $50 \mu \mathrm{m}$.

rostrocaudal extent of the caudate-putamen at $1 \mathrm{~mm}$ intervals (Fig. 2) was selected from the brain atlas of Paxinos and Watson (1982). In each rat, 6 sections matched to these standard sections were selected from a series of coronal sections stained for ChAT and SOM, respectively. In each section, counts were made of neurons labeled for only ChAT or SOM and those labeled both immunohistochemically and with ${ }^{3} \mathrm{H}$ thymidine. Counting was conducted unilaterally in the striatum, as delineated in Figure 2, in a microscope at a magnification of $250 \times$ with the aid of a grid. In the 2 most rostral sections representing the precommissural part of the striatum, the striatum was further divided into lateral and medial aspects (Fig. 4). No attempt was made to do the same in more caudal sections because of the more complex shape of the caudal striatum.

A striatal neuron was considered immunolabeled and counted if (1) its cytoplasm was stained with dark brown reaction product and (2) its nucleus was clearly visible. The criterion used for ${ }^{3} \mathrm{H}$-thymidine labeling was a minimum of 27 and 20 autoradiographic grains, respectively, for ChAT- and SOM-immunoreactive neurons. These criteria were established by the same procedure as in our previous study (Semba and Fibiger, 1988), and they allowed the inclusion of only heavily labeled neurons in the respective cell populations. No analyses were conducted on immunoreactive neurons that were lightly labeled with ${ }^{3} \mathrm{H}$-thymidine. Following cell counting, the percentage of double-labeled neurons out of all immunoreactive neurons was obtained for each antigen, and at each of the 6 rostrocaudal levels (and the lateral and medial regions for the 2 most rostral levels) for each gestational day of ${ }^{3} \mathrm{H}$-thymidine injection.

Two methodological issues related to the size difference between ChATand SOM-immunoreactive neurons (Fig. 1) should be noted here. First, the empirically established criteria for ${ }^{3} \mathrm{H}$-thymidine labeling were different for the 2 cell populations (see above). The most likely explanation for this difference is that, although the amount of incorporated ${ }^{3} \mathrm{H}$ thymidine in individual replicating cells would be the same for the 2 populations, the resolution of autoradiographic grains was better for the larger nuclei of ChAT-immunoreactive neurons because individual grains may overlap with one another more frequently in the smaller nuclei of the SOM neurons. [See also Appleton et al. (1969) for a discussion of the effects of nuclear size and shape on the detection of incorporated ${ }^{3} \mathrm{H}$-thymidine.]

Second, as shown in Figures 2-4, the percentage of double labeling was generally lower for SOM- than for ChAT-immunoreactive neurons. Again, the most likely explanation is that in SOM-labeled neurons, because of their smaller somal diameter, there would be more falsenegatives for ${ }^{3} \mathrm{H}$-thymidine labeling than in ChAT-immunoreactive neurons. This is because, although the antibodies used appeared to penetrate the $30 \mu \mathrm{m}$ sections completely, $\beta$ particles emitted by tritium cannot travel more than 1-2 $\mu \mathrm{m}$ through the tissue or emulsion (Hendrickson and Edwards, 1978). It follows, therefore, that for ${ }^{3} \mathrm{H}$-thymidine to be detected, the nucleus must essentially be in direct contact with the emulsion, and this should occur less frequently for SOM- than ChAT-labeled neurons because the generally smaller nuclei of SOMpositive neurons would more often be completely contained within the depth of the section.

\section{Results}

ChAT- and SOM-immunoreactive neurons were distributed homogeneously throughout the striatum. Examples of ${ }^{3} \mathrm{H}$-thymidine labeling in ChAT- and SOM-immunoreactive neurons are shown in Figure 1.

Figure 2 shows the percentage of double-labeled neurons among all ChAT- or all SOM-immunoreactive striatal neurons as a function of the embryonic day of ${ }^{3} \mathrm{H}$-thymidine injection. Double labeling of the ChAT-immunoreactive neurons peaked on E12 at the most caudal level of the striatum. More rostrally, the peak of double labeling occurred increasingly later, up to E15 at the most rostral level. The peak was also broader at more rostral levels. In contrast, the peak of double labeling of the SOM-immunoreactive neurons occurred sharply on E15 and E16 regardless of the rostrocaudal level.

The overall pattern of double labeling in the whole striatum 

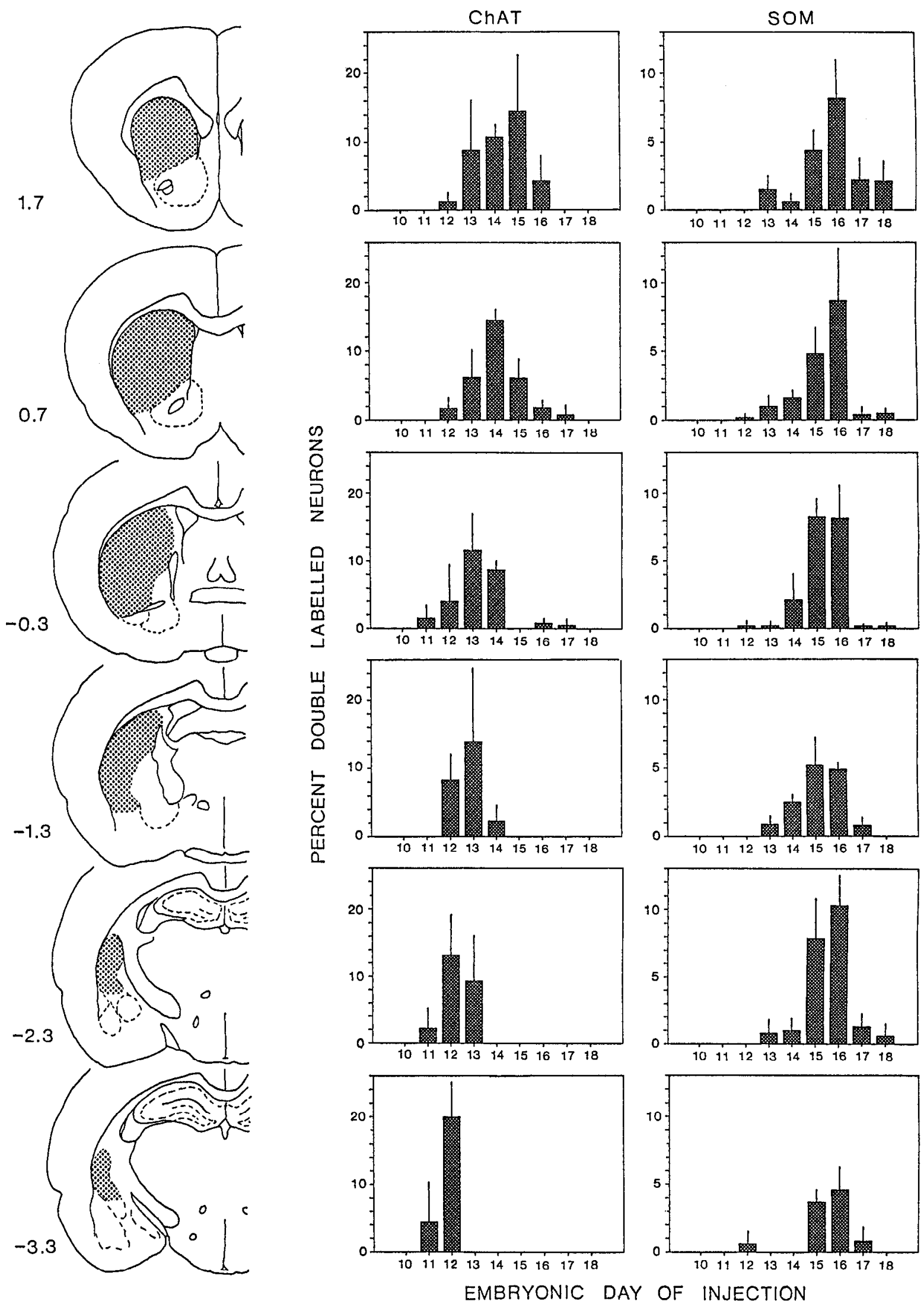

Figure 2. Percentage of neurons double-labeled for ChAT or SOM and ${ }^{3} \mathrm{H}$-thymidine among all ChAT- or all SOM-immunoreactive neurons as a function of embryonic day of ${ }^{3} \mathrm{H}$-thymidine injection at 6 different levels in the striatum. Each column in the graphs represents a mean of $2-4$ rats, and the bar on top of each column indicates SD. The 6 levels of the striatum used for analyses are shown at left; the number near each section drawing represents the distance from the bregma (in $\mathrm{mm}$ ), and stippling delineates the areas where cell counts were made. Note the caudal-torostral shift in the time of peak double labeling for the ChAT-positive neurons and the absence of such shift for the SOM-positive neurons. 


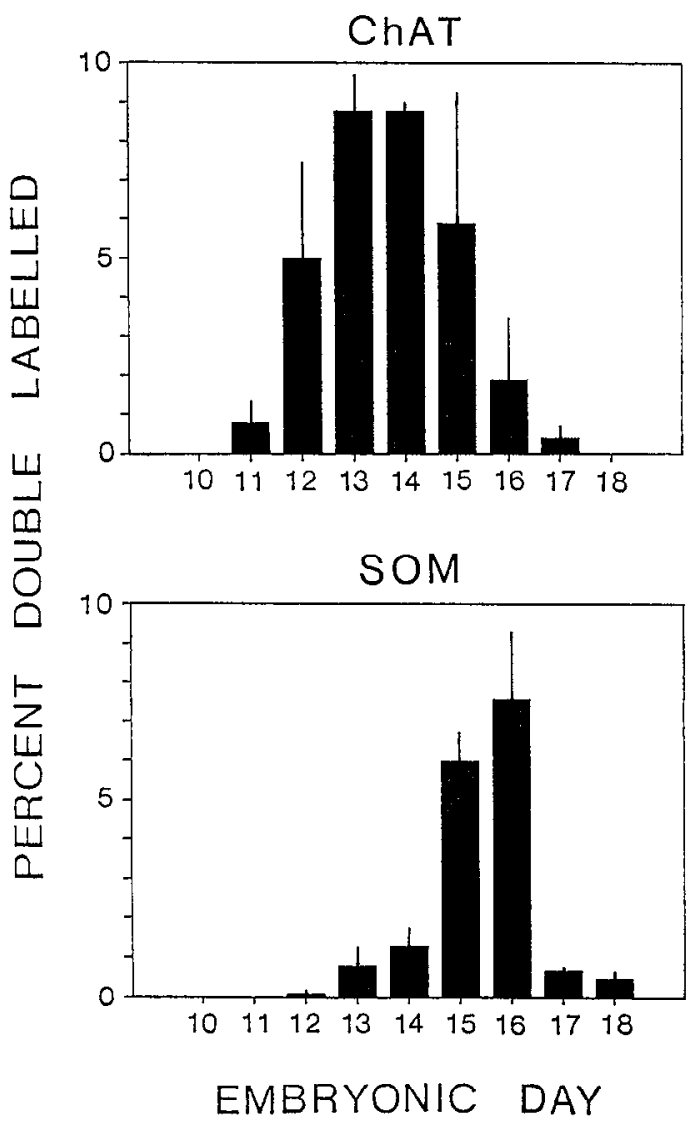

Figure 3. Percentage of neurons double-labeled for ChAT or SOM and ${ }^{3} \mathrm{H}$-thymidine of all ChAT- or all SOM-positive neurons in the entire striatum as a function of embryonic day of ${ }^{3} \mathrm{H}$-thymidine injection. The data presented in this figure were obtained by combining the data for all 6 levels of the striatum shown in Figure 2. The bar on top of each column represents SD.

was also different between ChAT- and SOM-immunoreactive neurons (Fig. 3). The peak of double labeling with ChAT-immunoreactive neurons was broad, encompassing days E12-15 and also E16 to some extent. The double labeling with SOMimmunoreactive neurons occurred later, and with a narrower peak, on E15-16.

To examine for possible mediolateral differences in the percentage of double labeling of the ChAT and SOM neurons, cell counts had been made separately for the lateral and the medial aspects of the striatum at the 2 most rostral levels (Fig. 4). With ChAT-positive neurons, the double labeling in the lateral aspect appeared to precede that in the medial aspect by about a day, although the difference was not statistically significant ( $\chi^{2}$ test). No obvious differences between the lateral and medial aspects were seen with SOM-immunoreactive neurons.

Figure 5 illustrates, in a 3-dimensional format, the overall spatiotemporal patterns of double labeling for ChAT- and SOMimmunopositive neurons, which are based on the data presented in Figure 2.

\section{Discussion}

The present data indicate that 2 major populations of interneurons in the striatum display patterns of neurogenesis that are distinctly different in terms of timing and topography. The neurogenesis of cholinergic neurons progresses in a caudal to rostral fashion from E12 to 15, whereas SOM-containing neurons throughout the striatum become postmitotic on about E15-16, i.e., towards the end of cholinergic neurogenesis. A more subtle, lateral-to-medial gradient is also present with cholinergic neurons in the rostral striatum, whereas no such gradient is apparent with SOM-containing neurons.

\section{Temporal relations of final mitosis and neurotransmitter phenotype expression}

The expression of neurotransmitter phenotype is an important aspect of neuronal differentiation. ChAT activity has been reported to be detectable on E13.5 in the developing striatum (Kessler, 1986), 1-2 d after final mitosis of the earliest developing cholinergic neurons. This is among the earliest reported day of detection of any neurotransmitter-related chemical in the striatum. Despite the early signs of acetylcholine synthesis, however, striatal cholinergic markers including ChAT and acetylcholinc activitics, cholinc uptakc, and muscarinic receptor binding remain relatively low even during the first postnatal week, and major increases occur during the following 3 weeks (McGeer et al., 1971; Coyle and Yamamura, 1976).

SOM in the striatum has been biochemically detected on E15 in the rat (Kessler, 1986), and this corresponds to the early phase of the peak final mitosis of SOM-containing neurons observed in the present study. Immunohistochemically, however, SOMcontaining neurons have not been detected until E1 8-19 (sperm positivity, E1) in the rat striatum (Shiosaka et al., 1982). Neurons immunoreactive for neuropeptide $Y$, which colocalizes with SOM in the adult striatum (Vincent et al., 1982; Smith and Parent, 1986), have also first been detected on E18 in the rat (Woodhams et al., 1985).

\section{Patterns of neurogenesis in the striatum}

Previous studies using ${ }^{3} \mathrm{H}$-thymidine autoradiography and Nissl staining in the rat have reported that striatal neurons generally undergo final mitosis from E12 to as late as postnatal day 2, with a peak around E15 (Fentress et al., 1981; Bayer, 1984; Marchand and Lajoie, 1986). Hence, the cholinergic neurons appear to be among the first neurons in the striatum to become postmitotic, whereas the SOM-containing neurons leave the mitotic cycle at about the time of peak striatal neurogenesis. The caudal-to-rostral gradient in cholinergic neurogenesis is consistent with previous findings for large striatal neurons (Bayer, 1984; Marchand and Lajoie, 1986), which is not surprising since most large neurons in the striatum observed in Nissl-stained material are cholinergic interneurons (Graybiel and Ragsdale, 1983; Parent, 1986). Early neurogenesis of striatal cholinergic neurons, apparently followed by the early onset of acetylcholine synthesis, raises the possibility that these neurons influence the development of later-developing neurons in the striatum.

The pattern of neurogenesis of medium-sized neurons observed in Nissl-stained material is more complex. Although specific descriptions vary among different studies, these neurons appear to be generated between E12 and postnatal day 2, with a slight caudal-to-rostral gradient at least in the precommissural striatum and with a medial-to-lateral gradient in the caudal striatum but a lateral-to-medial gradient in the rostral striatum (Fentress et al., 1981; Bayer, 1984; Marchand and Lajoie, 1986). Clusters of isochronically generated medium-sized neurons have been seen in the rostral striatum (Graybiel and Hickey, 1982; Marchand and Lajoie, 1986; van der Kooy and Fishell, 1987; also see below). One likely explanation for the complex pattern and prolonged period of neurogenesis is the heterogeneity that 


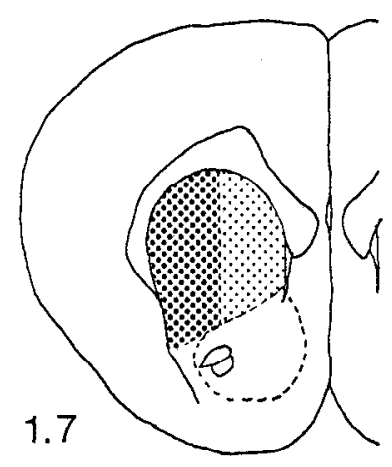

ChAT

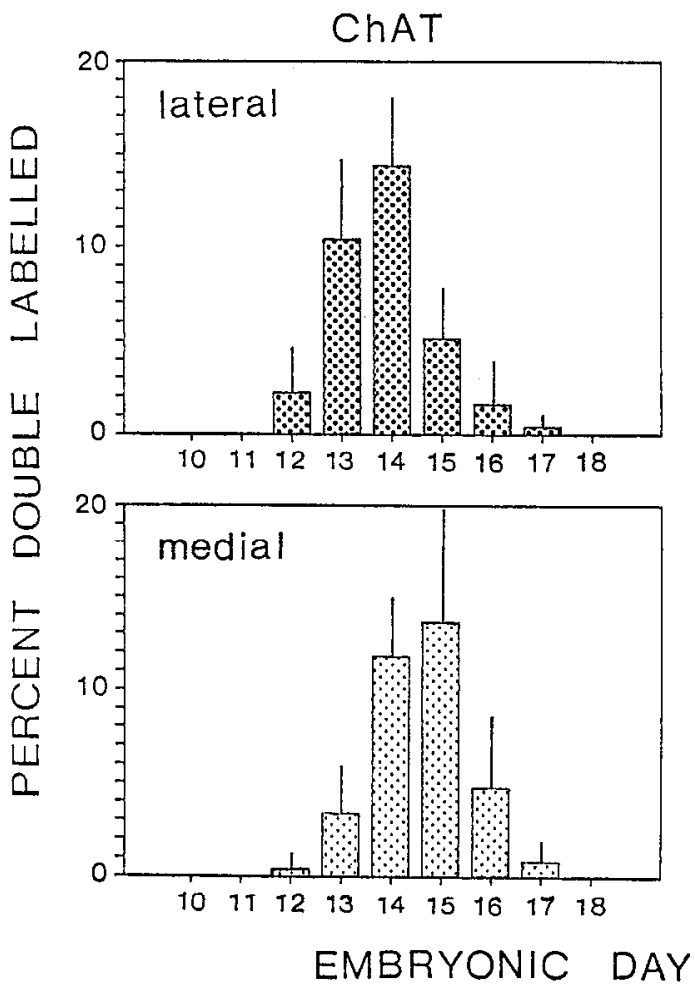

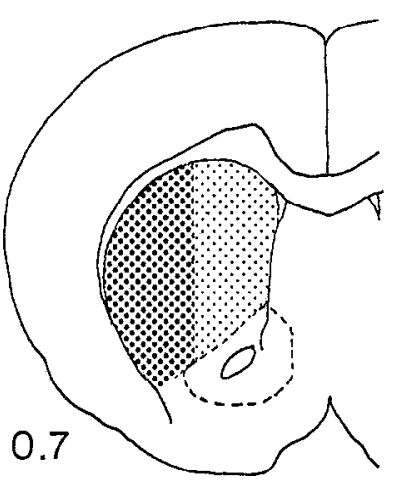
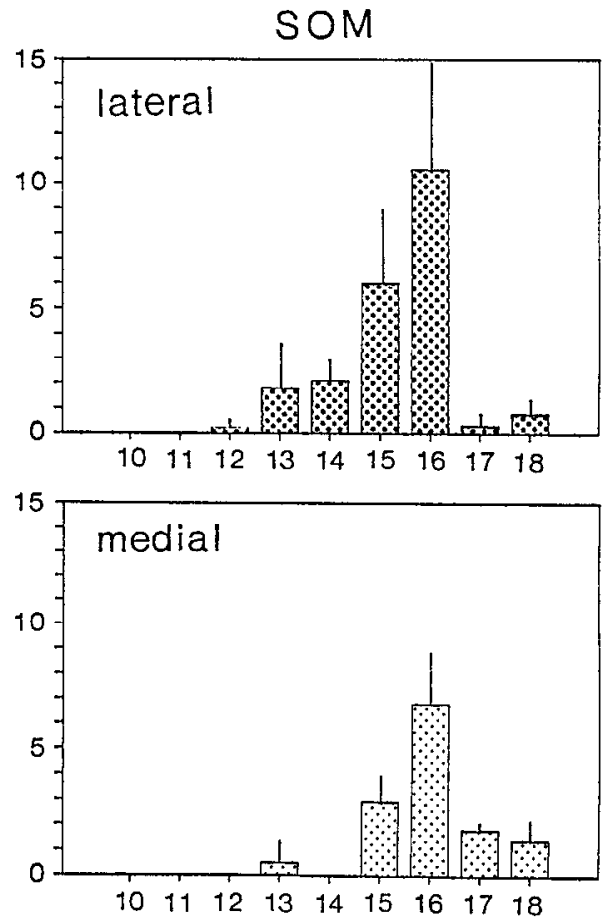

INJECTION
Figure 4. Percentage of neurons double-labeled for ChAT or SOM and ${ }^{3} \mathrm{H}$ thymidine of all ChAT- or all SOMpositive neurons in the lateral (heavy stippling) and medial (light stippling) aspects of the precommissural striatum, i.e., the 2 most rostral levels (1.7 and $0.7 \mathrm{~mm}$ rostral to the bregma) shown in Figure 2. exists among medium-sized striatal neurons in terms of their projections and neurotransmitter content (Graybiel and Ragsdale, 1983; Parent, 1986; Semba et al., 1987). This pattern possibly represents a composite of separate patterns displayed by different subpopulations of medium-sized neurons. This hypothesis is supported by the present findings with SOM-containing neurons; although these neurons are medium in sizc, they display a pattern of neurogenesis that is clearly distinct from that of medium-sized striatal neurons in general.

A unique characteristic of the neuronal organization in the striatum is the presence of mosaic patterns in which various anatomical markers are localized to macroscopic "patches" against the background "matrix" (see reviews by Graybiel and Ragsdale, 1983; Gerfen, 1987; Semba et al., 1987; van der Kooy et al., 1987). Both cholinergic (Graybiel et al., 1986) and SOMcontaining perikarya (Gerfen, 1984; Chesselet and Graybiel, 1986) have been reported to be distributed more or less homogeneously in relation to these compartments, although cholinergic and SOM-immunoreactive fibers appear to be more concentrated in the matrix. In the present study, no attempt was made to analyze the data in relation to compartmental organization. However, in the rat, most neurons that become postmitotic on E13-15 are found in opiate-receptor-rich "patches," whereas those leaving the mitotic cycle later, on E1820 , tend to be found in the opiate receptor-poor matrix (van der Kooy and Fishell, 1987; see also Graybiel and Hickey, 1982; Marchand and Lajoie, 1986). It appears, therefore, that cholinergic and SOM-containing neurons leave the mitotic cycle at about the same time as the majority of patch neurons and clearly before the most matrix neurons undergo their final mitosis. Since patches occupy only about $15 \%$ of the total area in sections of the adult striatum (Lança et al., 1986), a question remains as to the process by which these early-developing cholinergic and SOM-containing neurons eventually become evenly distributed between the 2 compartments.

A subtle lateral-to-medial gradient was seen in the present study in the neurogenesis of cholinergic neurons in the precommissural striatum. Similar lateromedial gradients have been observed in the concentration of muscarinic receptor binding in the striatum of rat, feline, and human fetuses (Nastuk and Gray- 

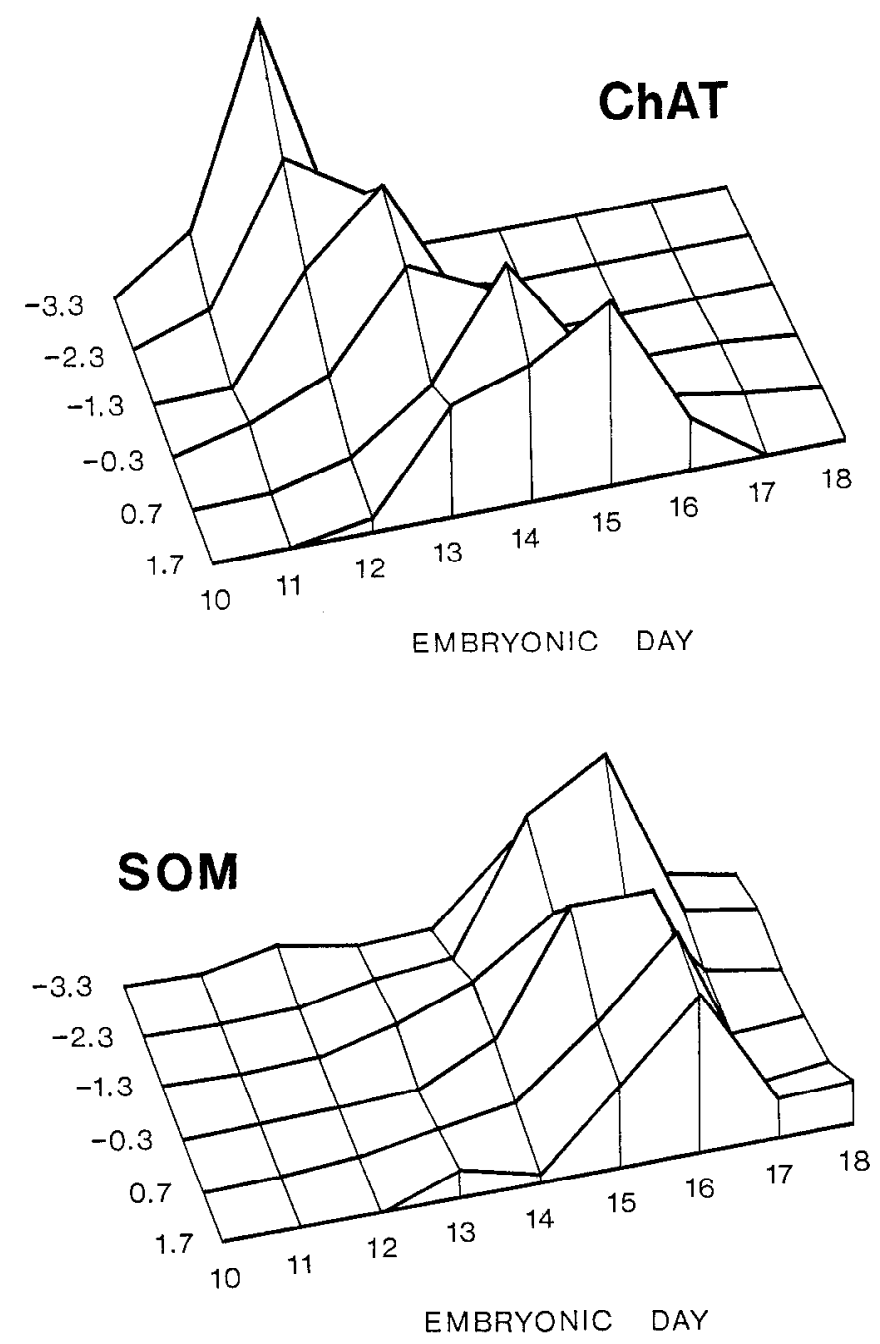

Figure 5. Spatiotemporal patterns of the percentage double labeling with ChAT or SOM and ${ }^{3} \mathrm{H}$-thymidine among all ChAT- and all SOMimmunoreactive neurons as a function of embryonic day of ${ }^{3} \mathrm{H}$-thymidine exposure. The numbers at left represent distances from bregma at 6 longitudinal levels of the striatum as shown in Figure 2. The display was constructed by using the data shown in Figure 2.

biel, 1985). There is evidence that the lateromedial difference in cholinergic activity continues into early postnatal life, and in part even into the adulthood. In perinatal rats and mice, the intensity of acetylcholinesterase staining of neuropil (Butcher and Hodge, 1976; Höhmann and Ebner, 1985) and perikarya following DFP treatment (J. Lehmann, S. Atmadja, and H. C. Fibiger, unpublished observations) is greater in the lateral than in the medial striatum. Sodium-dependent high-affinity choline uptake is greater in the lateral than in the medial striatum of newborn and juvenile baboons (Lowenstein et al., 1987). In the adult rat, the magnitudes of cholinergic markers including acetylcholine, muscarinic receptor binding, and high-affinity choline uptake are greater in the lateral than in the medial striatum (Guyenet et al., 1977; Takano et al., 1980; Rea and Simon, 1981; Strong et al., 1982; but see Fonnum et al., 1978). Although the present data indicate that SOM-containing neurons undergo final mitosis at about the same time, SOM-immunoreactive neurons appear first in the lateral striatum (Shiosaka et al., 1982), and similar observations have been reported with neurons immunoreactive for neuropeptide Y (Woodhams et al.,
1985), which colocalizes with SOM in the adult striatum (Vincent et al., 1982; Smith and Parent, 1986). Taken together, these observations suggest that the lateral-to-medial gradient may be a general organizational principle of the embryonic and, to some extent, the adult striatum.

\section{Relationship between the patterns of neurogenesis and striatal morphogenesis}

Neurogenesis is an integral part of morphogenesis, and the spatiotemporal pattern of neurogenesis must be understood in light of the mechanisms of cell proliferation and migration. In fact, gradients of neurogenesis are often taken to indicate that the oldest neurons are located farthest from the site of origin and the youngest near the site of origin (e.g., Bayer and Altman, 1987). How do the gradients of cholinergic neurogenesis and the apparent lack of gradients in SOM neurogenesis fit into the general scheme of striatal morphogenesis?

Embryologically, the striatum derives from the 2 ganglionic eminences, i.e., 2 elevations in the germinal epithelium of the developing forebrain (Hewitt, 1958; Smart, 1976; Smart and Sturrock, 1979; Lammers et al., 1980; Fentress et al., 1981). Of the 2, the medial eminence appears first, at about E11 in the mouse, the species in which striatal morphogenesis has been best studied (gestation period, $19 \mathrm{~d}$ ), and a day later the lateral eminence emerges rostral and dorsal to the medial eminence. Starting caudally the 2 cmincnces subsequently fuse, and by E1 6 they lose separate identities. The formation of the embryonic striatum is based on active cell proliferation, which occurs first in the ventricular zone (E11-13 in the mouse) and thereafter in the subventricular zone (Smart, 1976).

In light of the morphogenic process of the striatum, it is possible that the caudal-to-rostral gradient of cholinergic neurogenesis is generated during the process in which the lateral eminence appears subsequent and rostral to the medial eminence. Furthermore, the lateral-to-medial cholinergic gradient seen in the precommissural striatum is probably related to the migration of postmitotic neurons from the ventricular zone to the more laterally located, primordial striatum (Smart, 1976); as more cells are generated, younger neurons settle in more medial regions, thus forming lateral-to-medial gradient. It is, however, not clear what mechanisms account for the apparent lack of gradients in any direction in the neurogenesis of SOMcontaining neurons. Because these neurons are generated later, they most likely undergo their final mitosis in the subventricular zone, and these postmitotic neurons must somehow settle eventually in their final locations, which are homogeneously distributed throughout the striatum.

The formation of the striatum does not occur as an isolated event, but occurs as an integral part of the morphogenic process of the entire forebrain. It is therefore interesting to note that the spatiotemporal pattern of striatal cholinergic neurogenesis observed in the present study closely parallels that of the cholinergic neurons in the basal forebrain, which provide the major extrinsic cholinergic input to the cerebral cortex (Semba and Fibiger, 1988). These findings suggest that, despite their difference in projection seen in the adult, during ontogeny these 2 types of cholinergic neuron might arise from the same germinal source. In the adult rat, the 2 cholinergic cell populations are coextensive with each other along the longitudinal axis, with the striatal cholinergic neurons lying lateral to the basal forebrain cholinergic neurons (Schwaber et al., 1987). Cholinergic neurons in fetal striatal tissue cocultured with hippocampal slices 
develop physiologically functional projections into the hippocampal tissue (Gähwiler and Hefti, 1985). Taken together, these findings suggest that cholinergic neurons in the striatum and basal forebrain may share a common germinal source and that the expression of hodological phenotype in these neurons may be primarily regulated by extrinsic factors.

\section{References}

Angevine, J. B., Jr., and J. A. McConnell (1974) Time of origin of striatal neurons in the mouse: An autoradiographic study. Anat. Rec. 178: 300 .

Appleton, T. C., S. R. Pelc, and M. H. Tarbit (1969) Formation and loss of DNA in intestinal epithelium. J. Cell Science 5: 45-55.

Bayer, S. A. (1984) Neurogenesis in the rat neostriatum. Int. J. Dev. Neurosci. 2: 163-175.

Bayer, S. A., and J. Altman (1987) Directions in neurogenetic gradients and patterns of anatomical connections in the telencephalon. Prog. Neurobiol. 29: 57-106.

Bolam, J. P., B. H. Wainer, and A. D. Smith (1984) Characterization of cholinergic neurons in the rat neostriatum. A combination of choline acctyltransferase immunocytochemistry, Golgi-impregnation and electron microscopy. Neuroscience 12: 711-718.

Bolam, J. P., C. A. Ingham, P. N. Izzo, A. I. Levey, D. B. Rye, A. D. Smith, and B. H. Wainer (1986) Substance P-containing terminals in synaptic contact with cholinergic neurons in the neostriatum and basal forebrain: A double immunocytochemical study in the rat. Brain Res. 397: 279-289.

Brand, S., and P. Rakic (1979) Genesis of the primate neostriatum: $\left[{ }^{3} \mathrm{H}\right]$ thymidine autoradiographic analysis of the time of neuron origin in the rhesus monkey. Neuroscience 4: 767-778.

Butcher, L. L., and G. K. Hodge (1976) Postnatal development of acetylcholinesterase in the caudate-putamen nucleus and substantia nigra of rats. Brain Res. 106: 223-240.

Chesselet, M. F., and A. M. Graybiel (1986) Striatal neurons expressing somatostatin-like immunorcactivity: Evidence for a peptidergic interneuronal system in the cat. Neuroscience 17: 547-571.

Coyle, J. T., and H. I. Yamamura (1976) Neurochemical aspects of the ontogenesis of cholinergic neurons in the rat brain. Brain Res. 118: 429-440.

DiFiglia, M., and N. Aronin (1982) Ultrastructural features of immunoreactive somatostatin neurons in the rat caudate nucleus. $J$. Neurosci. 2: 1267-1274.

DiFiglia, M., and N. Aronin (1984) Quantitative electron microscopic study of immunoreative somatostatin axons in the rat neostriatum. Neurosci. Lett. 50: 325-331.

Fckenstein, F., and H. Thoenen (1982) Production of specific antisera and monoclonal antibodies to choline acetyltransferase: Characterization and use for identification of cholinergic neurons. EMBO J. 1: 363-368.

Fentress, J. C., B. B. Stanfield, and W. M. Cowan (1981) Observations on the development of the striatum in mice and rats. Anat. Embryol. 163: $275-298$.

Fernández, V., H. Bravo, R. Kuljis, and I. Fuentes (1979) Autoradiographic study of the development of the neostriatum in the rabbit. Brain Behav. Evol. 16: 113-128.

Fibiger, H. C. (1982) The organization and some projections of cholinergic neurons of the mammalian forebrain. Brain Res. Rev. 4:327388.

Fibiger, H. C., K. Semba, and S. R. Vincent (1987) Time of origin of immunohistochemically identified interneurons in the rat striatum. Soc. Neurosci. Abstr. 13: 1575.

Fonnum, F., Z. Gottesfeld, and I. Grofova (1978) Distribution of glutamate decarboxylase, choline acetyltransferase and aromatic amino acid decarboxylase in the basal ganglia of normal and operated rats. Evidence for striatopallidal, striatoentopeduncular and striatonigral GABAergic fibres. Brain Res. 143: 125-138.

Gähwiler, B. H., and F. Hefti (1985) Striatal acetylcholinesterasecontaining interneurons innervate hippocampal tissue in co-cultured slices. Brain Kes. 18: 311-314.

Gerfen, C. R. (1984) The neostriatal mosaic: Compartmentalization of corticostriatal input and striatonigral output systems. Nature 311 : $461-464$.

Gerfen, C. R. (1987) The neostriatal mosaic: Compartmental organization of mesostriatal systems. In The Basal Ganglia. II. Structure and Function-Current Concepts, M. B. Carpenter and A. Jayaraman, eds., pp. 65-80, Plenum, New York.

Graybiel, A. M., and T. L. Hickey (1982) Chemospecificity of ontogenetic units in the striatum: Demonstration by combining $\left[{ }^{3} \mathrm{H}\right]$ thymidine neuronography and histochemical staining. Proc. Natl. Acad. Sci. USA 79: 198-202.

Graybiel, A. M., and C. W. Ragsdale, Jr. (1983) Biochemical anatomy of the striatum. In Chemical Neuroanatomy, P. C. Emson, ed., pp. 427-503, Raven, New York.

Graybiel, A. M., R. W. Baughman, and F. Eckenstein (1986) Cholinergic neuropil of the striatum observes striosomal boundaries. Nature 323: 625-627.

Guyenet, P., C. Euvrard, F. Javoy, A. Herbet, and J. Glowinski (1977) Regional differences in the sensitivity of cholinergic neurons to dopaminergic drugs and quipazine in the rat striatum. Brain Res. 136: $487-500$.

Hendrickson, A., and S. B. Edwards (1978) The use of axonal transport for autoradiographic tracing of pathways in the central nervous system. In Methods in Physiological Psychology, Vol. 2, Neuroanatomical Research Techniques, R. T. Robertson, ed., pp. 241-290, Academic, New York.

Hewitt, W. (1958) The development of the human caudate and amygdaloid nuclei. J. Anat. (Lond.) 92: 377.

Höhmann, C. F., and F. F. Ebner (1985) Development of cholinergic markers in mouse forebrain. I. Choline acetyltransferase enzyme activity and acetylcholinesterase histochemistry. Dev. Brain Res. 23: 225-241.

Hsu, S.-M., L. Raine, and H. Fanger (1981) Use of avidin-biotinperoxidase complex (ABC) in immunoperoxidase techniques: A comparison between $\mathrm{ABC}$ and unlabeled antibody (PAP) procedures. J. Histochem. Cytochem. 29:577-580.

Kessler, J. A. (1986) Differential regulation of cholinergic and peptidergic development in the rat striatum in culture. Dev. Biol. 113: 7789.

Kubota, Y., S. Inagaki, S. Shimada, S. Kito, F. Eckenstein, and M. Tohyama (1987) Neostriatal cholinergic neurons receive direct synaptic inputs from dopaminergic axons. Brain Res. 413: 179-184.

Lammers, G. J., A. A. M. Gribnau, and H. J. ten Donkelaar (1980) Neurogenesis in the basal forebrain in the Chinese hamster (Cricetulus griseus). II. Site of neuron origin: Morphogenesis of the ventricular ridges. Anat. Embryol. 158: 193-211.

Lança, A. J., S. Boyd, B. E. Kolb, and D. van der Kooy (1986) The development of a patchy organization of the rat striatum. Dev. Brain Res. 27:1-10.

Lowenstein, P. R., P. A. Slesinger, H. S. Singer, L. C. Walker, M. F. Casanova, D. L. Price, and J. T. Coyle (1987) An autoradiographic study of the development of $\left[{ }^{3} \mathrm{H}\right]$ hemicholinium- 3 binding sites in human and baboon basal ganglia: A marker for the sodium-dependent high affinity choline uptake system. Dev. Brain Res. 34: 291-297.

Marchand, R., and L. Lajoie (1986) Histogenesis of the striopallidal systcm in the rat. Neurogenesis of its neurons. Neuroscience 17:573590.

McGeer, E. G., H. C. Fibiger, and V. Wickson (1971) Differential development of caudate enzymes in the neonatal rat. Brain Res. 32: 433-440.

Nastuk, M. A., and A. M. Graybiel (1985) Patterns of muscarinic cholinergic binding in the striatum and their relation to dopamine islands and striosomes. J. Comp. Neurol. 237: 176-194.

Parent, A. (1986) Comparative Neurobiology of the Basal Ganglia. Wiley, New York.

Paxinos, G., and C. Watson (1982) The Rat Brain in Stereotaxic Coordinates, Academic, New York.

Phelps, P. E., C. R. Houser, and J. E. Vaughn (1985) Immunocytochemical localization of choline acetyltransferase within the rat neostriatum: A correlated light and clcctron microscopic study of cholinergic neurons and synapses. J. Comp. Neurol. 238: 286-307.

Rea, M. A., and J. R. Simon (1981) Regional distribution of cholinergic parameters within the rat striatum. Brain Res. 219: 317-326.

Satoh, K., W. A. Staines, S. Atmadja, and H. C. Fibiger (1983) Ultrastructural observations of the cholinergic neuron in the rat striatum as identified by acetylcholinesterase pharmacohistochemistry. Neuroscience 10: 1121-1136.

Schwaber, J. S., W. T. Rogers, K. Satoh, and H. C. Fibiger (1987) Distribution and organization of cholinergic neurons in the rat forebrain demonstrated by computer-aided data acquisition and threedimensional reconstruction. J. Comp. Neurol. 263: 309-325. 
Semba, K., and H. C. Fibiger (1988) Time of origin of cholinergic neurons in the rat basal forebrain. J. Comp. Neurol. 269: 87-95.

Semba, K., S. R. Vincent, and H. C. Fibiger (1986) Time of origin of immunohistochemically identified interneurons in the rat striatum. A paper presented at the International Basal Ganglia Society Second Triennial Symposium, Victoria, British Columbia, Canada.

Semba, K., H. C. Fibiger, and S. R. Vincent (1987) Neurotransmitters in the mammalian striatum: Neuronal circuits and heterogeneity. Can. J. Neurol. Sci. 14: 386-394.

Shiosaka, S., K. Takatsuki, M. Sakanaka, S. Inagaki, H. Takagi, E. Senba, Y. Kawai, H. Iida, H. Minagawa, Y. Hara, T. Matsuzaki, and M. Tohyama (1982) Ontogeny of somatostatin-containing neuron system of the rat: Immunohistochemical analysis. II. Forebrain and diencephalon. J. Comp. Neurol. 204: 21 1-224.

Smart, I. H. M. (1976) A pilot study of cell production by the ganglionic eminences of the developing mouse brain. J. Anat. 121: 71-84.

Smart, I. H. M., and R. R. Sturrock (1979) Ontogeny of the neostriatum. In The Neostriatum, I. Divac and R. G. E. Öberg, eds., pp. 127146, Pergamon, Oxford.

Smith, Y., and A. Parent (1986) Neuropeptide Y-immunoreactive neurons in the striatum of cat and monkey: Morphological characteristics, intrinsic organization and co-localization with somatostatin. Brain Res. 372: 241-252.

Strong, R., T. Samorajski, and Z. Gottesfeld (1982) Regional mapping of neostriatal neurotransmitter systems as a function of aging. J. Neurochem. 39: 831-836.

Takagi, H., P. Somogyi, J. Somogyi, and A. D. Smith (1983) Fine structural studies on a type of somatostatin-immunoreactive neuron and its synaptic connections in the rat neostriatum: A correlated light and electron microscopic study. J. Comp. Neurol. 214: 1-16.

Takano, Y., Y. Kohjimoto, K. Uchimura, and H.-O. Kamiya (1980)
Mapping of the distribution of high affinity choline uptake and choline acetyltranferase in the striatum. Brain Res. 194: 583-587.

ten Donkelaar, H. J., and P. J. W. Dederen (1979) Neurogenesis in the basal forebrain of the Chinese hamster (Cricetulus griseus). I. Time of neuron origin. Anat. Embryol. 156: 331-348.

van der Kooy, D., and G. Fishell (1987) Neuronal birthdate underlies the development of striatal compartments. Brain Res. 401: 155-161.

van der Kooy, D., G. Fishell, L. A. Krushel, and J. G. Johnston (1987) The development of striatal compartments: From proliferation to patches. In The Basal Ganglia. II. Structure and Function-Current Concepts, M. B. Carpenter and A. Jayaraman, eds., pp. 81-98, Plenum, New York.

Vincent, S. R., L. Skirboll, T. Hökfelt, O. Johansson, J. M. Lundberg, R. P. Elde, L. Terenius, and J. Kimmel (1982) Coexistence of somatostatin- and avian pancreatic polypeptide (APP)-like immunoreactivity in some forebrain neurons. Neuroscience $7: 439-446$.

Vincent, S. R., O. Johansson, T. Hökfelt, L. Skirboll, R. P. Elde, L. Terenius, J. Kimmel, and M. Goldstein (1983a) NADPH-diaphorase: A selective histochemical marker for striatal neurons containing both somatostatin- and avian pancreatic polypeptide (APP)-like immunoreactivities. J. Comp. Neurol. 217: 264-270.

Vincent, S. R., W. A. Staines, and H. C. Fibiger (1983b) Histochemical demonstration of separate populations of somatostatin and cholinergic neurons in the rat striatum. Neurosci. Lett. 35: 111-114.

Vincent, S. R., C. H. S. McIntosh, A. M. J. Buchan, and J. C. Brown (1985) Central somatostatin systems revealed with monoclonal antibodies. J. Comp. Neurol. 238: 169-186.

Woodhams, P. L., Y. S. Allen, J. McGovern, J. M. Allen, S. R. Bloom, R. Balazs, and J. M. Polak (1985) Immunohistochemical analysis of the early ontogeny of the neuropeptide $Y$ system in rat brain. Neuroscience 15: 173-202. 